and this hospital will stand as an everlasting memorial of their philanthropy. It is a child born out of charity and entirely devoted to charity.

The following are the names of the ophthalmic surgeons in charge of the Sir Cowasji Jehangir Ophthalmic Hospital, Bombay, since the year it came into existence, namely 1866 :-

1866 to July, 1867.--Surgeon-General Sir William Guyer Hunter, M.D., F.R.C.S.E.

July, 1867 to January, 1872.-Major J. H. Sylvester, M.R.C.S., F.G.C.

January 28, 1872 to May 31, 1895.-Brigade Surgeon Lt.-Col. George Archibald Maconachie, M.D., C.M.(Aber.).

June 1, 1895 to April 19, 1907.-Lt.-Col. H. Herbert, I.M.S.

April 20, 1907 to February 28, 1914.-Lt.-Col. P. P. Kilkelly, M.B., I.M.S.

March 1, 1914 to December 31, 1922.--Lt.-Col. G. McPherson, M.A., M.B., C.M., F.R.C.S.E., I.M.S., C.I.E.

January 1, 1922 onwards.-Lt.-Col. Sir J. N. Duggan, Kt., C.I.E., O.B.E., D.O. (Oxon), F.C.P.S., L.M. \& S.

\title{
REFERENCES
}

1. History of the Indian Medical Service, 1600-1913. By Lt.-Col. D. G. Crawford, Vol. II, pp. 391-400.

2. Grant Medical College Annul Reports, 1845-1862.

\section{MICROPHTHALMIA AND THE VISUAL PATHWAYS A Case Associated with Blindness and Imbecility, and Sex-linked}

BY

S. E. Whitnall and R. M. Norman

BURDEN MENTAL RESEARCH TRUST, STOKE PARK COLONY, BRISTOL

IN this investigation the chief points-beyond the history and observations of the patient during life-are the gross and microscopical features of the eyeballs; then of the visual pathways, traced along the optic nerves, chiasma and tracts to the lower visual centres in the midbrain; finally the brain as a whole, and the cortical visual areas in particular, were examined.

(The remarkable and possibly unique feature of this eye condition being a recessive sex-linked one has already been the subject of a paper by Dr. Fraser Roberts (1937), the genealogy showing 14 cases, all males, traced in four generations.) 
The bulk of the literature on microphthalmia is generally concerned with clinical observations on the living, or examination of the eyeballs after death; and considerations of any effects of such malformation on the visual pathways to the brain and in its visual cortical areas seem rare. Hence this investigation, which is particularly directed towards the latter, may be worthy of record.

We would preface, however, with the note that since the eyeballs in the present case were found completely disorganised, undeveloped, and obviously incapable of receiving or transforming light, the cerebral effects may be expected comparable to those of anophthalmia (congenital or experimentally produced), where they have been studied and to a vast extent, though with confusing and contradictory results.

Without a tedious and inconclusive analysis of detail, such records seem to come into two broad groups. Namely, the one where the visual cortex is found structurally altered, and the other where it is found to be little, if at all, affected. In both groups there have been described decided changes from the normal in the lower visual centres (the lateral geniculate and superior quadrigeminal bodies) as well as in the optic nerves, chiasma and tracts. And generally it may be said that the visual cortex appears to be less affected by experiments on lower animals, than is found by clinical observations upon man.

Of the former group of records of alteration in the cortex, Leonowa (1893, 1896) especially found the lamina granulosa absent, and he considers that this cell layer and the stria of Gennari are only developed when the cortical visual area is functionally active. Some support to this is given by Hanke; by Bolton and Campbell, who found marked reduction in thickness of the stria in cases of old-standing total blindness; and by Monakow, who found the visual system atrophied all the way through in a congenitally blind man 75 years old. Hippel (1909) (in Schwalbe's monograph of bodily malformations in general), states that in anophthalmus congenitus, there frequently occurs an absence of the optic nerves, chiasma and tracts, a reduction of the pulvinar, with changes in the cellular structure of the visual cortex.

On the other hand, Woollard (1926) in a case of anophthalmus congenitus in a new-born puppy found no alteration in the cellular lamination of the visual cortex; nor did Monakow and Michel, after experiments on various animals. Tsang (1937) gives a good review of the literature concerning both clinical and experimental observations (the above undated citations are taken from his paper and from that by Woollard). Tsang experimented on rats; and after enucleation of the eyeballs at birth examined the effects at three subsequent stages of growth, and found the 
time factor was decisive in inducing degeneration of the visual centres; the superior quadrigeminal bodies were most affected, next the lateral geniculate bodies, and least of all the cortical visual areas. He suggests that this gradation of effects is due to the different functional intimacy of the retinal fibres with these visual centres. He also remarks, as possibly explaining the variant results of experiments, that there were different methods used and upon quite different animals, in which the visual system plays very different roles in life.

The present case of what must have been complete blindness from birth to death at 17 years, presented a combination of features differing from those noted above. There were completely malformed and functionless eyeballs; ribbon-like optic nerves; a small chiasma and thread-like tracts; also a reduction in size of the lateral geniculate bodies, with defective lamination. But the superior quadrigeminal bodies were normal in size and structure, the optic radiation was well formed, and the visual cortex, though reduced in surface area, showed a well-marked stria of Gennari and lamina granulosa, with little cortical cellular alteration elsewhere. These conditions were found in both hemispheres, though, as will be described, the right occipital lobe was malformed.

The case comes under van Duyse's (1899) third group of microphthalmia - those without coloboma but associated with other abnormalities; and Ida Mann's (1936, 1937) third classification of eye-defects-an arrest of development plus a superimposed aberrance. The cause most likely is an inherent fault in the germ plasm. It may be added that the eyeballs may be small but otherwise normal (Parsons, 1904). Also that several writers have observed that microphthalmia can be associated with mental impairment.

The particulars of our case now follow.

History.-The patient (serial number D.115, in the records) was admitted to Stoke Park Colony, Bristol, on October 11, 1935, and died on September 9, 1937. He was a congenitally blind imbecile of a chronological age of 17 years and 4 months at death. He was the third of four male children; the youngest is also blind and mentally defective, but the parents and two elder boys are normal in all ways.

Examination.- He could hear, had a limited vocabulary but no conversation; knew the names of common objects, could distinguish and count his fingers, but was dull, stupid and vacant. He had unsteady gait, was excitable, and restlessly rocked his body; he required care, supervision and control, but was of clean habits. Height 5 feet $2 \frac{1}{2}$ inches, weight 6 st. $2 \frac{1}{2}$ lbs.; emaciated; 
his skeletal and bodily abnormalities were general physical undevelopment, with a narrow subcostal trunk, genu valgum, and short terminal phalanges of the thumbs.

His years of retardation from the normal were:-in head size (1283 c.c.) five years; stature standing and sitting, each a year late; weight two years below average for age. The Binet intelligence tests indicated a mental age of four years, giving an I.Q. of 29. But owing to the blindness this figure is only a rough estimate. He walked and started talking at approximately two years of age.

He died of acute broncho-pneumonia. The post-mortem examination showed the skull to be of normal thickness, but with inequality of the two halves, the right being smaller, especially in the occipital region; there were no other points of particular interest.

The eyes, in life, were examined by Mr. Iles, of Bristol, whose report states that both were sunken in the sockets; intra-ocular tension minus; no nystagmus. The cornea of the right eye

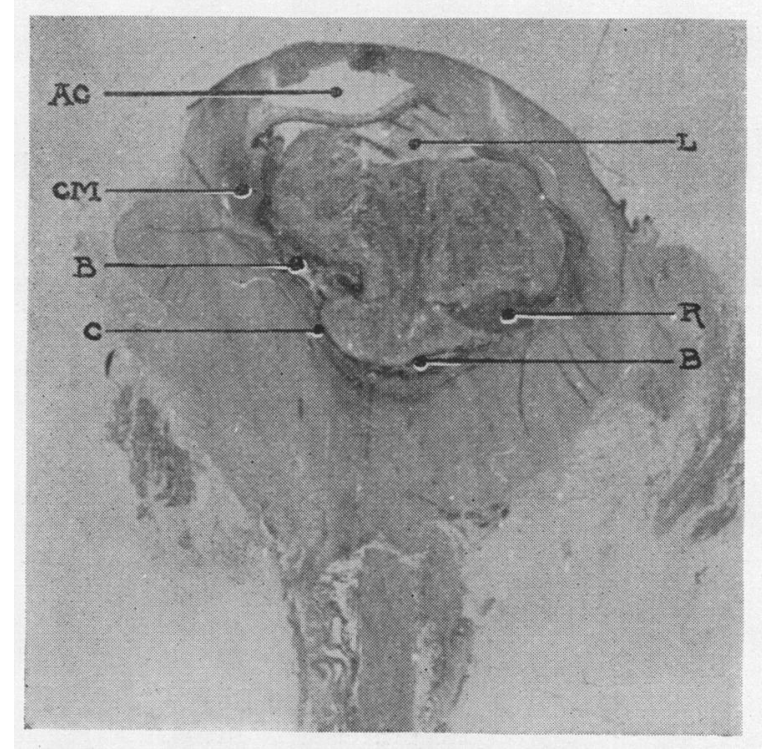

Fig. 1.

Micro-section through right eye of D.115. Note enormously thickened cornea and sclera; partial obliteration of anterior chamber (A.C. with iris behind); lens rudimentary (L); retina quite undifferentiated, but producing a mass of neuroglial and pigment epithelial proliferation (R.) filling the entire vitreous chamber, with bone formation behind it (B.). The ciliary muscle and process are seen (C.M.); also the thickened choroid (C.). The optic nerve is thickened here, just behind the globe, by neuroglia and dural sheath, but became ribbon-like towards the chiasma. $\times 3$. 
showed dense leucoma adherens and ground-glass opacity over a sector of about $60^{\circ}$; no vascularity; anterior chamber shallow, pupil drawn up and very small; lens not seen. The cornea of the left eye was opaque all over, with vessels spreading from the ciliary zone; iris adherent to cornea.

After death and preservation in formalin solution the eye-balls had transverse and antero-posterior dimensions of $15 \mathrm{~mm}$. each in the left eye, and of $13 \mathrm{~mm}$. each in the right one. (The normal size is about $24 \mathrm{~mm}$. in those dimensions; and according to the measurements of Weiss, the eyeballs in this case were smaller than those of the new-born, the same diameters of which are 16.0 and $16.4 \mathrm{~mm}$.). Unfortunately, it was not possible to obtain a correlation with the size of the orbits. The oblique muscles were apparently absent, but the recti were well developed.

A microscopical examination of the right eyeball was kindly made by Mr. Shapland of Moorfields Eye Hospital, through the introduction of Miss I. C. Mann. It was fixed in formalin, decalcified with nitric acid and phloroglucin, embedded in celloidin, stained with haematoxylin and eosin, then sectioned horizontally above (Fig. 1). The report is as follows :-

"Microscopic appearance:-The corneal epithelium is thinned and in places absent (artefact). Bowman's membrane shows numerous rucks and is in places completely destroyed. The substantia propria of the cornea is enormously thickened and fibrotic, and shows marginal deep vascularisation. Descemet's membrane is thickened and thrown into folds. Its endothelium is flattened. The anterior chamber is largely obliterated, there being a wide peripheral anterior synechia on each side. There are also anterior synechiae at the pupillary border of the iris on each side. The iris stroma shows a moderate infiltration with small round cells, and there is a considerable degree of atrophy of the pigment epithelium. There is ectropion uveae on each side. The lens is largely undeveloped, but the anterior lens capsule can be recognised lying curled up behind the iris in the pupillary area. The retina is quite undifferentiated and the seat of considerable neuroglial proliferation, which fills the entire vitreous chamber. There is considerable proliferation of the pigment epithelium posteriorly, and here it has formed columns and apparent tubules which give the appearance of a glandular epithelium. There is considerable formation of bone in this region and on the inner surface of the choroid. The ciliary muscle is well developed, but the ciliary processes are much flattened and elongated. The choroid is thickened and shows a considerable degree of fibrous tissue overgrowth. It also shows a moderate degree of small round cell infiltration. The lamina vitrea can be recognised in places as a corkscrew-like band of elastic tissue immediately external to the zone of proliferating 
pigment epithelium. The optic nerve shows neuroglial proliferation." (Moorfields report 108/op/37 of 3.3.38.)

The left eyeball likewise had normal recti muscles and the obliques seemed absent. Divided sagittally by us, it showed similarly an enormously thickened sclera, but within the globe there were present even greater abnormalities. The choroidal ossification was far more extensive and formed a cup-like

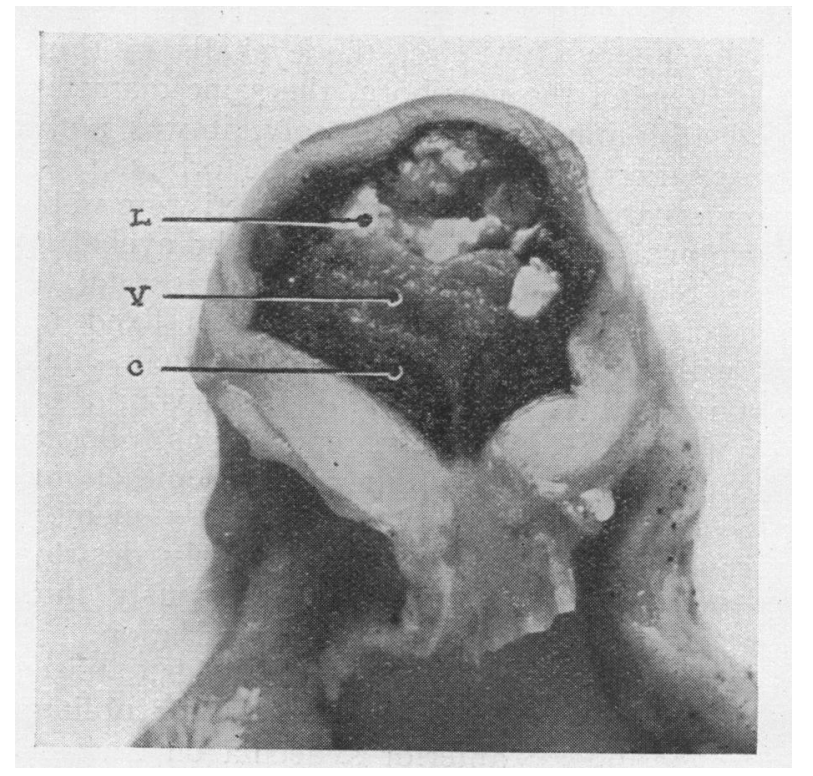

FIG. 2 .

Gross section of the left eye of D.115, sagittally cut. There is a similar thickening of the sclera. The choroid (C.) shows further extension of ossification. V. represents the vitreous, entering which behind is a persistent hyaloid artery. L. is a calcareous mass representing the lens, and obliterating the anterior chamber. $\times 3$.

thickening, within which lay a mass of proliferated tissue representing the vitreous (to which ran apparently a well-marked persistent hyaloid artery, well displayed in the figure). In front of this, a large calcareous mass represented the lens and obliterated completely the anterior chamber. There was also a large calcareous plaque in the vitreous, seen on the right side of the photograph. The ciliary bodies were prominent.

The optic nerves were large near the eyeballs, due to thickening of the sheath together with neuroglial proliferation, but became ribbon-like towards the chiasma, which was also greatly reduced in size. Only a few thin beaded myelinated fibres were present in the nerves. 


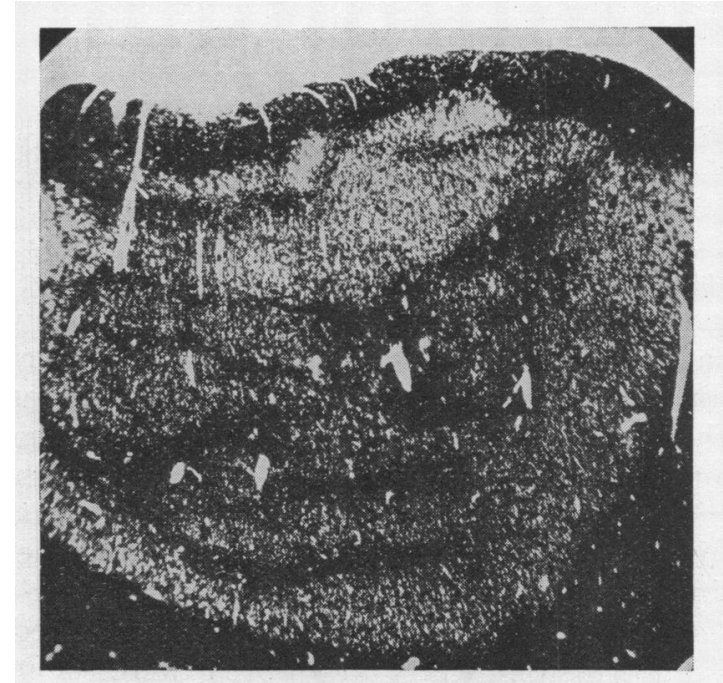

FIG. 3.

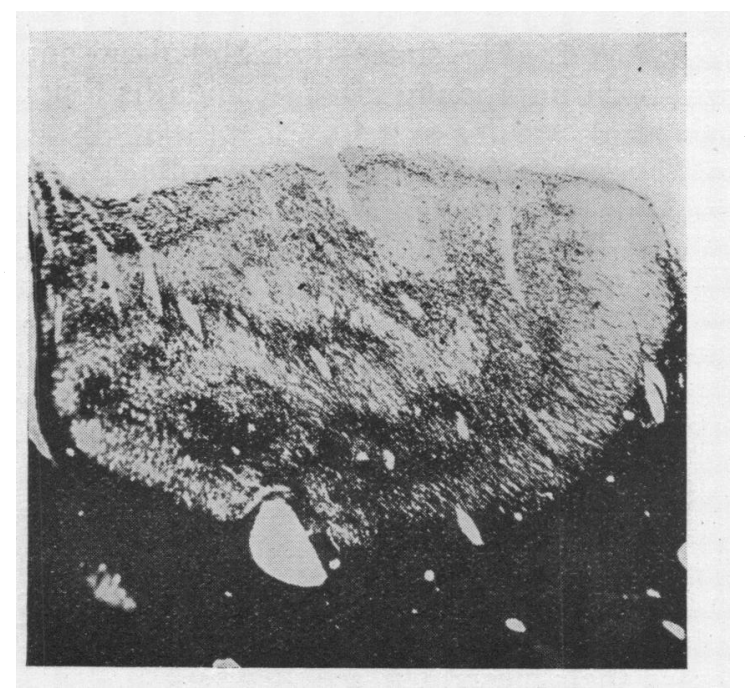

FIG. 4.

Microphotographic sections of the lateral geniculate bodies, showing differences in size and lamination between a normal subject (3) and the defective one (4). Stained Weigert-Pal method for myelin. $\times 13$. 
The optic tracts were mere threads, containing scattered myelinated fibres in a dense neuroglial matrix.

The lateral geniculate bodies.-On inspection it was obvious that these were considerably smaller than normal (about halfsize). On sectioning, the usual laminated appearance was seen to be grossly disturbed in both myelin and nerve-cell preparations. The number of finer myelinated fibres was obviously less than usual. The nerve cell bodies were not arranged in the normal evenly spaced-out state, but were small, reduced in numbers and patchily distributed throughout, and showed an abnormal variability in size. An increase in fibrous neuroglia was present in the left lateral geniculate as compared with a normal control (Holzer stain). There was no increase in the normal lipoid content of the nerve cells; in fact, owing to the small size and immature appearance of many of them, rather less than normal.

The superior quadrigeminal bodies were of the usual size, and showed no histological differences from the normal, the lamination and cells being regular (myelin and nerve-cell stains).

The left optic radiation was quite well-developed as traced throughout, and histologically its fibres were definitely fully myelinated.

It may be added for comparison that Woollard (1926), in his investigation of the anophthalmic puppy referred to above, found the optic nerves, chiasma and tracts were thread-like, but the pulvinar, lateral geniculate and superior quadrigeminal bodies were well developed. He states that Monakow enucleated both eyes in a new-born puppy and after six months found no changes at all in the central nervous system, except that all the fibres going to the occipital convolutions were non-medullated.

The brain, after injection with 10 per cent. formol saline through the orbits, was removed and suspended by the middle cerebral arteries in a bucket of the same fluid to avoid distortion during hardening. As a whole it showed no gross abnormality save a mongoloid flattening and reduction in size of the right occipital lobe (in harmony with the malformation of the skull). This character may be illustrated by its " tongue-ing " or backward projection (taken dioptrically from the mid-point of a line dropped vertically from the parieto-occipital sulcus to the base-line of the hemisphere, thence to the extreme pole) measuring only $2 \mathrm{~mm}$.; whereas in the average Mongol brain it was found to extend $8 \mathrm{~mm}$.; in the defective $10 \mathrm{~mm}$.; and in the normal $15 \mathrm{~mm}$., along similar lines on their mesial surface views.

The "Brain Product" (which is the simplest comparative index of its bulk, and estimated by multiplying together the length, breadth and heighth of the cerebrum, and taking the first three figures of the result) gave in this case a figure of 238 against an average normal one of 248 (in 78 cases), and an average 
defective of 203 (in 100 cases). The right hemisphere was $156 \mathrm{~mm}$. long, the left 160 , against a normal average of $169 \mathrm{~mm}$. and a defective average of $156 \mathrm{~mm}$. The cortical surface " receptor and effector areas " of Berry were within the planimeter dioptric normal ranges. The weight of the right hemisphere stripped and drained, was 528 grams, that of the left 546 , contrasting with a normal of 554 for each; or a total of 1,074 against 1,108 grams. for the cerebrum. Therefore, the brain of D.115 weighed less and was a little smaller than an average normal one; it was considerably larger, however, than those often found in imbeciles. The cerebellum and brain-stem were of normal dimensions.

The reduction in bulk of the right occipital lobe as compared with the normal (which can be seen in Figs. 5 and 6) was shown by devising an "occipital product" of three dimensions taken on the mesial surface of the sectioned brain : length (from just below splenium to extreme pole), height (from the parieto-occipital sulcus to lowest point of pole), and thickness (from just behind splenium to most prominent part of opposite surface, by calipers). The findings were :-

\begin{tabular}{cl|c|c|c}
\hline & $\begin{array}{c}\text { Whole } \\
\text { Hemispheric } \\
\text { Product }\end{array}$ & $\begin{array}{c}\text { Occipital } \\
\text { Product }\end{array}$ & $\begin{array}{c}\text { Percentage of } \\
\text { Occipital to } \\
\text { whole Product }\end{array}$ \\
\hline Average of & & & & \\
13 Normals. & R.H. & 123.2 & 17.5 & 14.2 \\
D.115 Right & H. & 119 & 10.6 & 8.9 \\
Left & H. & 119 & 11.6 & 9.7 \\
Difference & R.H. & 4.2 & 6.9 & 5.2 \\
& L.H. & 4.2 & 5.9 & 4.4 \\
\hline
\end{tabular}

The pattern of the cortical sulci and gyri was fairly normal, save for the following features: On the lateral aspect each central sulcus of Rolando only just reached the upper margin of the hemisphere; the right one simulated a bipartate condition by the presence below of a well-marked tri-radiate subcentral sulcus. The right occipital gyri were narrow, crowded and irregular, especially towards the pole, whereas those on the left were perfectly developed. The left temporal pattern was most irregular; the superior gyrus being broken about its centre, the anterior half tapering off behind, and the posterior half running into the middle gyrus; the inferior gyrus was badly marked and 
broken up. The insulae were well operculated on both sides. For the rest of these surfaces, nothing was evident that could not be counterparted in normal hemispheres.

On the mesial aspect of each hemisphere, apart from the visual area, there was little to note. The corpus callosum was slender in the middle but of normal area in section. The cingulate sulcus was normal on the left but irregular and did not reach the upper margin of the hemisphere on the right, a feature, however, not uncommon in our series of 100 normal brains.

The visual area.-The superficial or surface extent of this on the mesial aspect of the occipital lobes was measured by the perimeter on dioptric tracings. The areas taken included both visuo-sensory and visuo-psychic subdivisions, following the delineations of Elliot Smith and von Economo. By actual comparisons, and further illustrated as a percentage of the whole hemispheric area from this view, they show striking differences from the average findings in a group of normal brains. (Measurements in square inches) :-

\begin{tabular}{|c|c|c|c|}
\hline & Visual Area & Whole Area & Percentage \\
\hline \multirow{2}{*}{$\begin{array}{l}\text { Average of } 13 \\
\text { Normals. Right } H \text {. }\end{array}$} & & & \\
\hline & $3 \cdot 26$ & $21 \cdot 19$ & $15 \cdot 38$ \\
\hline \multirow{2}{*}{$\begin{array}{lll}\text { D.115. } & \text { Right } \mathrm{H} . \\
& \text { Left } \mathrm{H} .\end{array}$} & $1 \cdot 91$ & $20 \cdot 38$ & $9 \cdot 37$ \\
\hline & $1 \cdot 76$ & $20 \cdot 38$ & $8 \cdot 63$ \\
\hline \multirow{2}{*}{$\begin{array}{ll}\text { Difference } & \text { R.H. } \\
& \text { L.H. }\end{array}$} & $1 \cdot 35$ & $0 \cdot 81$ & $6 \cdot 01$ \\
\hline & $1 \cdot 50$ & $0 \cdot 81$ & $6 \cdot 75$ \\
\hline
\end{tabular}

The subdivisions of the area (again using Elliot Smith's mapping, confirmed by von Economo's cellular method) were, in square inches:-

$$
\begin{array}{lccccc}
\text { R.H. striate, } & 0.72, \quad \text { parastr. } & 0.74 & \text { and peristr. } & 0.45 \\
\text { L.H. }, \quad 0.82, \quad, \quad 0.80 & , & 0.14 \\
& \text { The whole right area }= & 1.92 .
\end{array}
$$

A normal one gave striate 1.52 ; para. 1.08 ; peri. 58 .

The whole $=3.18$; thus showing a marked reduction in the defective, even allowing for the smaller brain size.

The cuneus was small on the right, the posterior calcarine sulcus making a "cuneal angle" with the parieto-occipital sulcus of $74^{\circ}$, and on the left one of $75.5^{\circ}$. A normal, we found, is about $89^{\circ}$, but with a great range of variations. 


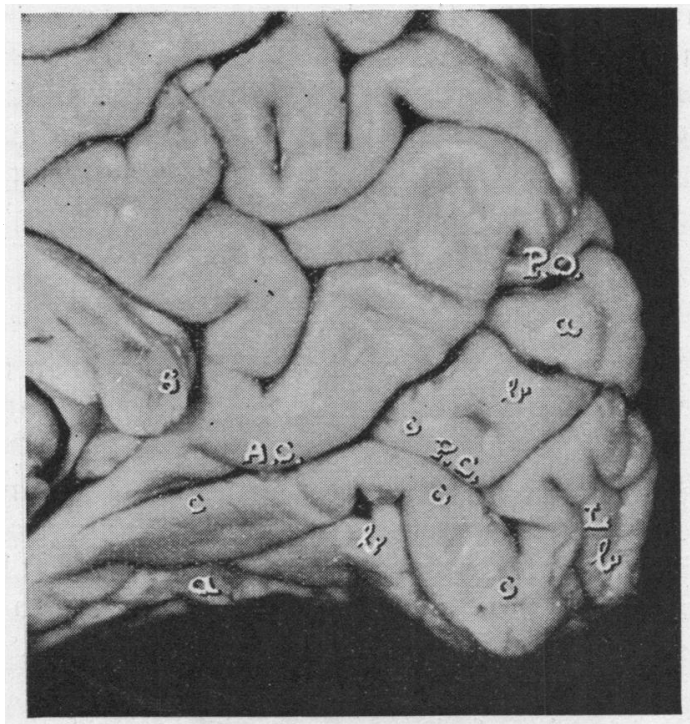

Fig. 5.

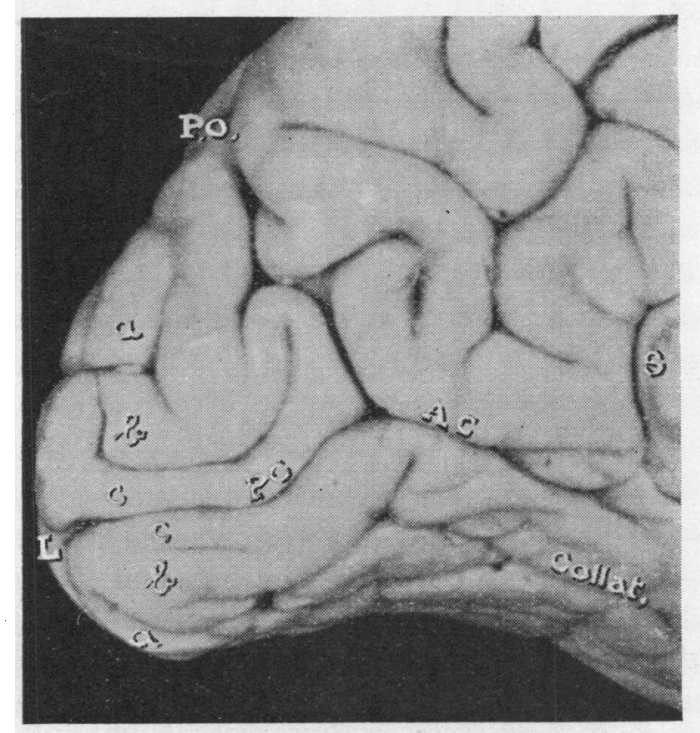

Fig. 6.

Cortical visual area maps of mesial surfaces of the occipital pole in the brain of D.115, right (5) and left (6) hemispheres. The $c, b, a$, areas are Economo's cellular markings, corresponding to the striate, para-striate, and peri-striate ones of Elliot Smith, and confirmed by histological sections. A.C. and P.C. are the limbs of the calcarine sulcus, and $L$. is the lunate sulcus. P.O. is the parieto-occipital sulcus, and S. the splenium. Natural size. 
The general pattern of the sulci in this region showed marked difference on the two sides. The left presented an almost perfect one (according to the mapping of Elliot Smith), there being a long posterior calcarine passing on to the pole and bifurcating against the roll of a lunate sulcus; there were superior (or limiting cuneal) and inferior (limiting lingual) shallower sulci, dividing superficially his striate area (the O.C. of von Economo) from the para-striate (O.B.); the peri-striate (O.A.) was marked from the latter by a transverse collateral sulcus. Above, the forward extent of the peri-striate area (O.A.) was as usual buried in the posterior wall of the parieto-occipital sulcus. These markings were supported by histological examination.

But on the right side there was a crumpling up and distortion of the whole area (though the microscope again gave verity to the more difficult interpretation of this map). The posterior calcarine was very short, and entirely confined to this aspect even with its forked ending, which was semicircled by a lunate sulcus, also on this surface.

The surface lengths of the component parts of these calcarine sulci, measured by a specially designed graduated wheel on dioptric tracings, may be of interest, if difficult of interpretation. The measurements in millimetres were:

\begin{tabular}{|c|c|c|c|c|c|}
\hline & & Ant. Calc. & Post. Calc. & Fork. & Total length. \\
\hline \multicolumn{6}{|c|}{$\begin{array}{l}\text { Normal } \\
\text { average of }\end{array}$} \\
\hline 57 & R.H. & $26 \cdot 3$ & $37 \cdot 9$ & $12 \cdot 7$ & $78 \cdot 7$ \\
\hline \multirow[t]{2}{*}{ D. 115} & R.H. & $28 \cdot 0$ & $18 \cdot 0$ & $6 \cdot 0$ & $52 \cdot 0$ \\
\hline & L.H. & $21 \cdot 0$ & $28 \cdot 0$ & $11 \cdot 0$ & $60 \cdot 0$ \\
\hline
\end{tabular}

indicating that the reduction in length was at the expense of the two later added components (the retinal macular ones) of this system, though, as noted below, there was compensation by greater depth on the right side. The right whole calcarine sulcus showed a more marked downward curve than the left. The depth of these calcarine sulci is of interest. That of the anterior calcarine taken in its centre was $7 \mathrm{~mm}$. in both hemispheres. In the left posterior calcarine, the depth was $12 \mathrm{~mm}$. but in the right it was $18 \mathrm{~mm}$., giving a compensation for its shorter surface appearance. There was no under-folding extent of the cortex at the bottom of the sulci. 


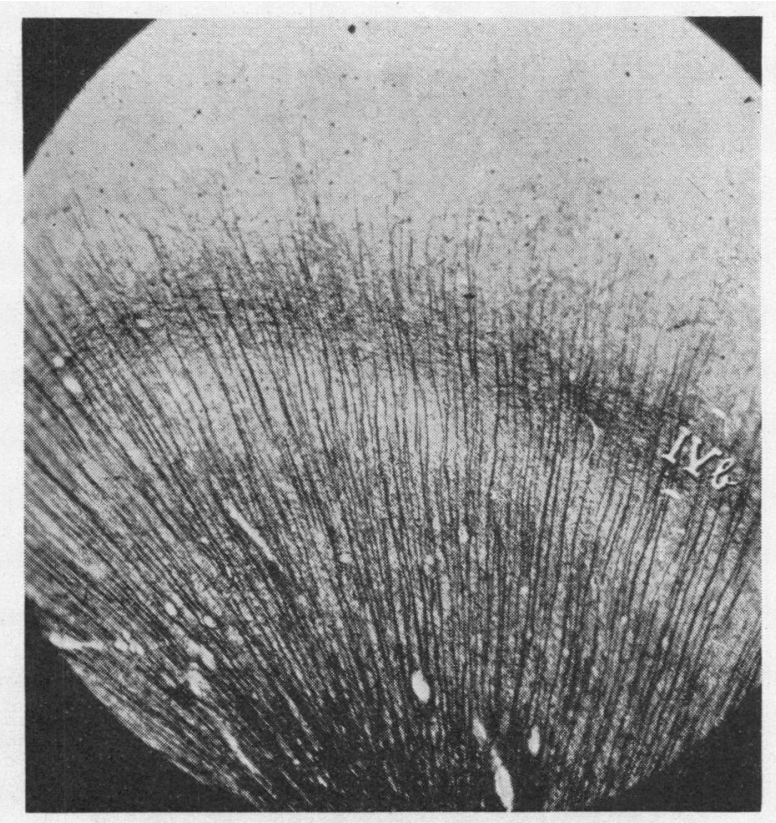

FIG. 7.

Showing the stria of Gennari (iv b.) present in the defective brain of D.115. (Section taken from about first third of the posterior calcarine sulcus. Stained for myelin.) $\times 40$.

The cortex, histologically examined, showed in general only those changes which are commonly found in the brains of mental defectives of no particular pathological type. In the para - and peristriate areas of Elliot Smith (areas $0 \mathrm{~B}$ and $0 \mathrm{~A}$ of von Economo) a slight irregularity in the arrangement of nerve cells was observed in the supra-granular layers, the normal well marked columnar arrangement being in places disturbed. The nerve cell bodies appeared unduly small and the Nissl's bodies stained poorly. This contrasted with the much better staining of sections taken from the parietal and frontal cortical areas. Myelin staining also showed no gross abnormality, a well-marked stria of Gennari being present and the normal transitions between the granular type of cortex (O.C.) and the para-striate type (O.B.) were constantly observed. The wealth of finer fibre content was, however, less than in normal controls. Stains for fibrous glia showed no abnormality. There was no appreciable diminution in cortical depth of grey matter compared with the normal.

As regards the cortex elsewhere, in the frontal, parietal and temporal regions one would say that a change was seen similar 
to that found in other mentally defective brains of no special pathological type; namely, a slight irregularity in the arrangement of the pyramidal cells, a lack of uniformity in size, and a tendency for clumping together of poorly formed cells in the

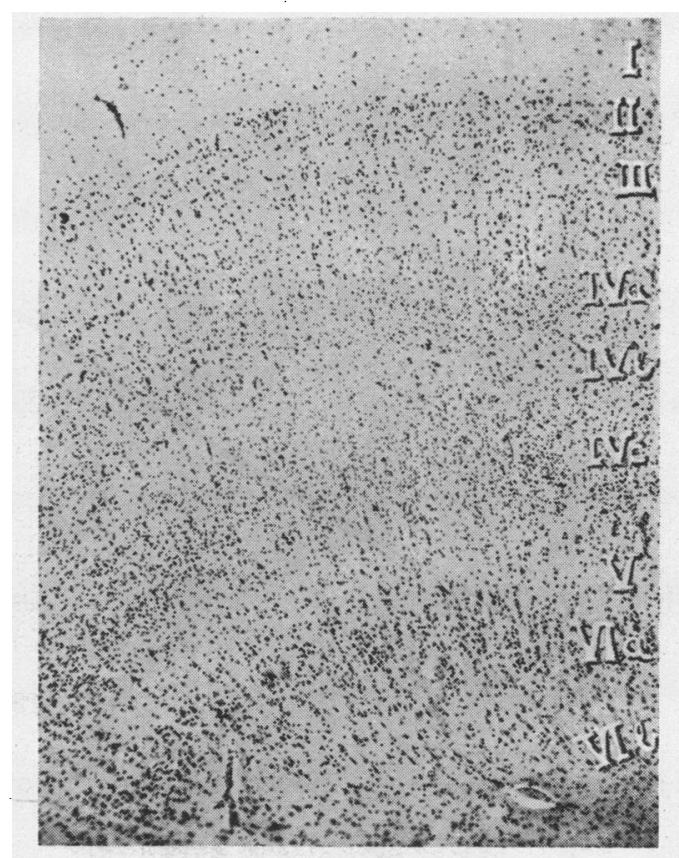

Fig. 8 .

View of the visual cortex in the same subject to show cell lamination. (Taken from same region as Fig. 7. Stained with cresyl-violet.) $\times 60$.

supra-granular layer. In some sections the pyramidal cells seemed reduced in number, but never to the gross extent seen in cortical atrophy. The cortex of the angular gyrus, described as the " centre" for " word-blindness," among other functions, showed no special configurative or cellular abnormality.

\section{Comments}

It is difficult to distinguish the effects of two different factors making for abnormality in this brain, the first being the general one of mental deficiency, the second the specific factor of lack of stimulation of the visual areas by impulses from the malformed eyes. In the main, it may be said that all the minor abnormalities found could be paralleled by other imbecile cortices in which peripheral blindness had played no part in the aetiology of the 
mental defect. A slight suggestion of specific inferiority of the visual areas was given by the fact that the nerve cells in these areas appeared less well developed than those of other parts of the same cortex, but it is impossible to rule out purely technical reasons for such an impression.

In the lateral geniculate bodies, on the other hand, abnormal changes were much more obvious. In addition to their poor development as a whole, there was evidence of atrophy (areas of reduced numbers of cells and gliosis). It is well known that optic atrophy may give rise to trans-synaptic degeneration of the nerve cells of these bodies. In this case, where a combination of simple development defect and degenerative pathological changes have both occurred in the eyes, it would seem probable that a somewhat similar condition has arisen in these nuclei, the end result being a disturbance of their normal structure (faulty lamination), coupled with mild pathological changes of a secondary character. It is presumably owing to the comparatively slight extent of the latter process that such insignificant changes were present in the visual areas.

The atrophy of the lower pathways in the optic nerves, chiasma and tracts again might in development be expected from their deprivation of light impulses. But as regards the normal appearance and composition of the superior quadrigeminal bodies, a retinal pathway to them is now denied by some authorities, and here is obviously quite absent; one may therefore conjecture whether the fibres which these bodies receive from the visual cortex through the optic radiations, from the spino-tectal tracts, medial lemniscus, and other sources, do not convey sufficient stimuli, should such be necessary, for their full development.

The gross malformation of the one right occipital lobe must be considered as adventitious and merely concomitant with the imbecility; and, in view of the other defects in this brain, could not have been the real or sole cause of the mental impairment, which is to be sought, so far as present methods avail, in the diffuse slight irregularities present in the cortex.

It is to be added as regards this investigation, that the general dimensions of the types of brains were supplied by the records of the large series of normal (100) and defective (nearly 200) ones in the Stoke Park Collection. But particular measurements and comparisons were made on the same series specially for this article, and they form one part only of an extensive range of studies thereon, as yet unpublished. This part of the work has been aided by a grant from the Colston Research Fund of Bristol, which is hereby gratefully acknowledged. And an appreciative note should be made of the services of the technical Assistant at Stoke Park, Mr. H. D. Steele. 


\section{Summary}

(1) A microphthalmic imbecile 17 years of age, with a unique ophthalmological family history, presented at autopsy special features in his eyeballs and visual tracts.

(2) The eyeballs were completely disorganised and could never have transmitted light-equivalent to an anophthalmic condition.

(3) The effects of this deprivation of light impulses were reflected in a pronounced poorness of development of the optic nerves, chiasma, tracts and lateral geniculate bodies.

But beyond this region :-

(4) The superior quadrigeminal bodies were quite normal in size and structure.

(5) The optic radiation was well formed and its fibres were myelinated.

(6) The cortical visual areas, though their surface extent was much less than usual and one was grossly deformed, presented a normal lamina granulosa and a well marked stria of Gennari.

(7) The imbecile condition is possibly explained by an imperfect general cortical cellular composition; for there were no gross cerebral pathological lesions present.

\section{BIBLIOGRAPHY}

Duyse, van.-" de l'Anophthalmie Congenitale." Arch. d'Ophtal., Vol. XIX, 1899.

Leonowa.-(i) (Anophthalmus and atrophy of the bulb). Arch. f. Anat. u. Entwick., 1893.

(ii) (Congenital Anophthalmus and atrophy of bulb in new-born children). Arch. f. Psychiat. u. Nervenkr., Vol. XXVII, p. 53, 1896.

MANN, IDA-(i) "Developmental Abnormalities of the Eye." 1937. Cambridge University Press, Also

(ii) Article in "The Eye and its Diseases," edited by ConRAD BERENS. W. B. Saunders Co., p. 320, 1936.

Parsons.- "The Pathology of the Eye," Microphthalmia and Anophthalmia, Vol. III, p. 872, 1904.

Roberts, J. A. Fraser.- " Sex-linked Microphthalmia sometimes associated with mental deficiency." Brit. Med. Jl., Vol. II, p. 1213, December 18, 1937.

SCHWALBE.-Article by Hippel on " Missbildungen des Auges" in " Morphologie der Missbildungen," etc., Teil III, Lief. 1, Abt. 2, Kap. I, pp. 20 and 24 , 1909.

TsANG, Yu chan.- "Visual Centres in blinded rats." Jl. Comp. Neurol., Vol. LXVI, p. 211, 1937 (gives reviews of experimental and clinical observations, with an extensive bibliography).

Woollard, H. H.- "Anophthalmus congenitus in a puppy." Brit. Jl. Ophthal., p. $131,1926$. 